\title{
Transport of Entanglement Through a Heisenberg-XY Spin Chain
}

\author{
V. Subrahmanyam ${ }^{(1)}$ and Arul Lakshminarayan ${ }^{(2)}$ \\ (1) Department of Physics, Indian Institute of Technology, Kanpur, 208016, India. \\ (2) Department of Physics, Indian Institute of Technology Madras, Chennai, 600036, India.
}

\begin{abstract}
The entanglement dynamics of spin chains is investigated using Heisenberg-XY spin Hamiltonian dynamics. The various measures of two-qubit entanglement are calculated analytically in the timeevolved state starting from initial states with no entanglement and exactly one pair of maximallyentangled qubits. The localizable entanglement between a pair of qubits at the end of chain captures the essential features of entanglement transport across the chain, and it displays the difference between an initial state with no entanglement and an initial state with one pair of maximallyentangled qubits.
\end{abstract}

Quantum entanglement in spin systems is an extensively-studied field in recent years, in the advent of growing realization that entanglement can be a resource for quantum information processing ${ }^{1,2}$. Within this general field, entanglement of spin- $\frac{1}{2}$ degrees of freedom, qubits, has been in focus for an obvious reason of their paramount importance for quantum computers, not to mention their well-known applicability in various condensed-matter systems, optics and other branches of physics. For a pure state of many qubits, quantum entanglement is a measure of how a subsystem is correlated to the rest of the system, which can be quantified by the von Neumann entropy of the reduced density matrix of the subsystem.

For a system of a large number of qubits, how different pairs of qubits are entangled in a pure state cannot easily be specified, even with known diagonal and off-diagonal correlation functions. There are many pairwise entanglement measures of pure quantum states of many qubits, as we shall discuss below. Essentially, any two-qubit entanglement measure depends on the reduced density matrix, $\rho_{i j}$ of the marked pair of qubits $i$ and $j$. The reduced density matrix is obtained from the original many-qubit pure state $|\psi\rangle$ through a partial trace over the rest of the qubits, $\rho_{i j}=\operatorname{Tr}_{[i j]}|\psi\rangle\langle\psi|$. Using the two-qubit basis $|00\rangle,|01\rangle,|10\rangle,|11\rangle$, the reduced density matrix has the form

$$
\rho_{i j}=\left(\begin{array}{cccc}
A_{i j} & E_{i j} & F_{i j} & G_{i j} \\
E_{i j}^{\star} & B_{i j} & H_{i j} & I_{i j} \\
F_{i j}^{\star} & H_{i j}^{\star} & C_{i j} & J_{i j} \\
G_{i j}^{\star} & I_{i j}^{\star} & J_{i j}^{\star} & D_{i j}
\end{array}\right)
$$

The two-qubit reduced density matrix above represents a mixed state in general, though we started with a pure many-qubit state. We can calculate von Neumann entropy from the eigenvalues of $\rho_{i j}$, which quantifies how these two sites are entangled with the rest of the system. However, a measure of entanglement between these two sites cannot be straightforwardly quantified, because of the mixed-state structure. In the above each of the matrix elements can be expressed as an expectation value of a two-qubit operator in the initial state $|\psi\rangle$. Using the Pauli operators for a qubit, $\sigma^{+}|0\rangle=|1\rangle, \sigma^{+}|1\rangle=$
$0, \sigma^{z}|0\rangle=-|0\rangle, \sigma^{z}|1\rangle=|1\rangle .$, for instance,

$$
H_{i j}=\left\langle 01\left|\rho_{i j}\right| 10\right\rangle=\left\langle\psi\left|\sigma_{i}^{+} \sigma_{j}^{-}\right| \psi\right\rangle,
$$

and similarly the other matrix elements are expectation values of appropriate operators. A good measure of impurity of the two-qubit state is $M_{i j}=1-\operatorname{Tr} \rho_{i j}^{2}$, which is zero if $\rho_{i j}$ represents a pure state of the given pair of qubits. In general the reduced density matrix represents a mixed state, giving a nonzero value for $M_{i j}$. The von Neumann entropy calculated as $-\operatorname{Tr} \rho_{i j} \log \rho_{i j}$ quantifies entanglement of this pair with the rest of the qubits. When $M_{i j}=0$, the reduced density matrix represents a pure state for the pair of qubits and the von Neumann entropy would be zero. For $M_{i j} \neq 0$, the von Neumann entropy will be nonzero, indicating an entanglement of the pair with the rest of the system, and possibly a mutual entanglement among the pair of qubits. The pairwise concurrence ${ }^{3}$ is a measure that quantifies the mutual entanglement of the marked pair of qubits. It carries the information of entanglement sharing between site $i$ and any other site. If the concurrence between $i$ and $j$ is one, would imply neither of these two sites is entangled with the rest of the system, and hence a pure-state assignment can be done to these sites. If the concurrence less than one, that would imply site $i$ is sharing entanglement with site $j$ and the rest of the system too. The concurrence measure ${ }^{3}$ is given as

$$
\mathcal{C}_{i j}=\max \left(0, \lambda_{1}^{1 / 2}-\lambda_{2}^{1 / 2}-\lambda_{3}^{1 / 2}-\lambda_{4}^{1 / 2}\right) .
$$

In the above $\lambda_{i}$ are the eigenvalues in decreasing order of the matrix $\rho_{i j} \hat{\rho}_{i j}$, where $\hat{\rho}_{i j}$ is the time-reversed matrix, $\hat{\rho}_{i j}=\sigma_{i}^{y} \otimes \sigma_{j}^{y} \rho_{i j}^{*} \sigma_{i}^{y} \otimes \sigma_{j}^{y}$. The above concurrence measure captures the essential information of mutual entanglement of the marked pair of qubits. However, it is not clear if a given value of the concurrence for a pair of qubits would imply that one can separate the pair of qubits in an entangled state, and use the entanglement as a resource operationally for quantum information processing.

The localizable entanglement (LE) between two qubits of a many-qubit state is the maximal amount of entanglement system that can be concentrated on the given pair of qubits, on an average, by doing a measurement on the rest of the qubits $\underline{\underline{4}}$. 
The LE between two qubits can be isolated, by an appropriate measurement on the rest of the qubits, and thus can be used as resource. Also, this has a direct bearing on the correlations present between the pair of qubit in the parent many-qubit state. The calculation of LE could be very tedious, as one has to search through every possible measurement basis for the rest of the qubits. However, there are useful upper and lower bounds on LE, that are calculable from the diagonal correlation functions alone ${ }^{4}$. Let us denote

$$
Q_{i j}^{x} \equiv\left\langle\sigma_{i}^{x} \sigma_{j}^{x}\right\rangle-\left\langle\sigma_{i}^{x}\right\rangle\left\langle\sigma_{j}^{x}\right\rangle,
$$

and similarly $Q_{i j}^{y}, Q_{i j}^{z}$ in terms of $y y$ and $z z$ correlation functions, and

$$
s_{i j}^{ \pm} \equiv\left(1 \pm\left\langle\sigma_{i}^{z} \sigma_{j}^{z}\right\rangle\right)^{2}-\left(\left\langle\sigma_{i}^{z}\right\rangle \pm\left\langle\sigma_{j}^{z}\right\rangle\right)^{2} .
$$

In terms of the above correlation functions, the bounds on LE are

$$
\max \left(\left|Q_{i j}^{x}\right|,\left|Q_{i j}^{y}\right|,\left|Q_{i j}^{z}\right|\right) \leq L E_{i j} \leq \frac{\sqrt{s_{i j}^{+}}+\sqrt{s_{i j}^{-}}}{2} .
$$

Now, each of the matrix elements of the reduced density matrix $\rho_{i j}$ involves correlation functions. After some manipulations using the properties of the Pauli matrices, and their expectation values in the initial state, we have $Q^{x}=2 \operatorname{Re}(H+G)-4 \operatorname{Re}(E+J) \operatorname{Re}(I+F), Q^{y}=$ $2 \operatorname{Re}(H-G)-4 \operatorname{Im}(E+J) \operatorname{Im}(I+F), Q^{z}=4(A D-B C)$, and $s_{i j}^{+}=16 A D, s_{i j}^{-}=16 B C$. Here the $i j$ subscripts have been suppressed for clarity.

The entanglement properties depend only on the structure of entanglement of a many-body state without reference to a Hamiltonian. However, here we would like to investigate the entanglement dynamics of a simple interacting spin systems on a lattice. Consider a spin- $\frac{1}{2}$ spin system, a nearest-neighbour anisotropic Heisenberg model in one dimension with a Hamiltonian

$$
H=\frac{K}{2} \sum_{i}\left(S_{i}^{+} S_{i+1}^{-}+\text {H.C. }\right)+\Delta S_{i}^{z} S_{i+1}^{z}-E_{0}
$$

where $S_{i}^{+}$is spin-raising operator at site $i, K$ is the exchange interaction strength, and $\Delta$ a measure of anisotropy. A constant, $E_{0}$ the energy of a state with all down spins or all up spins, has been added on for convenience. Working in a diagonal basis of $S_{i}^{z}$ for every site $i$, there are two states per site, viz., an up-spin state (denoted by $|1\rangle$ and a down-spin state denoted by $|0\rangle$ ). A many-spin state can be characterized by the number of down spins, as the total $S^{z}$ is a good quantum number. We confine ourselves here to spin systems in one dimension, as eigenstates are known in this case through Bethe-Ansatz, and are well studied.

The concurrence measure of entanglement in the eigenstates of the above model have been investigated ${ }^{5.6 .7}$, and the entanglement dynamics have been discussed ${ }^{8.9 .10}$. The localizable entanglement has been studied for the
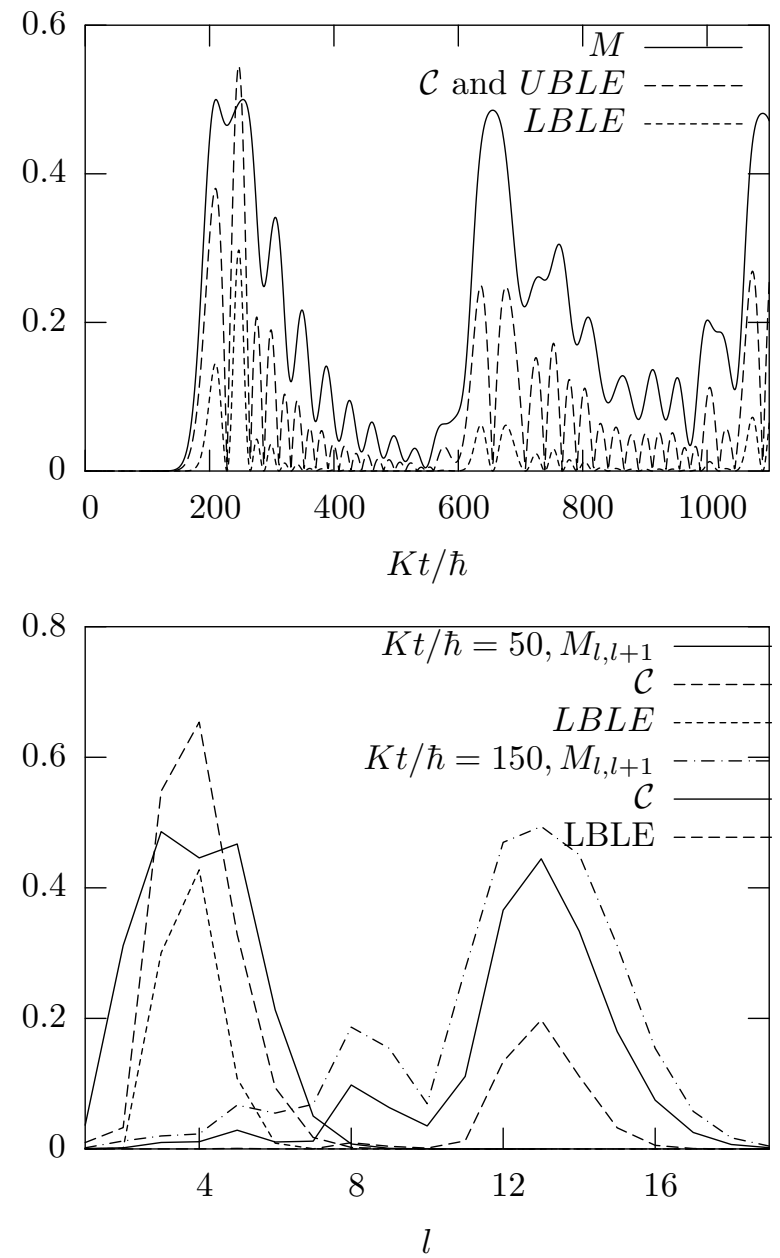

FIG. 1: The entanglement measures for the last pair of qubits in an unentangled initial state $\left|\psi_{u}\right\rangle$ are plotted vs. time, for a finite open chain of $N=20$ qubits. The upper bound on LE coincides with the concurrence measure in this sector with exactly on down spin. In the second figure the entanglement for nearest neighbour qubits $l, l+1$ are plotted vs $l$ for two different times $K t / \hbar=50,150$.

ground state ${ }^{11}$. In this study we will focus on the dynamics of the various entanglement measures for the above model. Our strategy is to start from a given initial state (either with no entangled pairs of qubits or with exactly one pair of qubits maximally entangled and the rest in an unentangled state), and study its entanglement properties using the measures discussed above, through the time evolution using Heisenberg dynamics. The initial state $|\psi(0)\rangle$, let us say with exactly one entangled pair of qubits at one end of the chain, will evolve into a state $|\psi(t)\rangle$ at a later time $t$, with a complicated distribution of entanglement, with dynamically changing correlation functions. We shall investigate how entanglement transport takes place across the spin chain, by studying the entanglement of a pair of qubits at the other end of the chain.

The dynamics of Heisenberg Hamiltonian above con- 
serves the number of down spins in a given state. This implies that, for an initial state with a definite number for down spins, at future times the reduced density matrix for a pair of qubits can have nonzero diagonal elements and a nonzero off-diagonal element $H_{i j}$ in Eq. (1), and we have

$$
E_{i j}=F_{i j}=G_{i j}=I_{i j}=J_{i j}=0
$$

which simplifies the expressions for the entanglement measures substantially. We have for a many-qubit state with a definite number of down spins (number of qubits in state $|0\rangle)$,

$$
\begin{array}{rc}
M_{i j}= & 1-A_{i j}^{2}-B_{i j}^{2}-C_{i j}^{2}-D_{i j}^{2}-2\left|H_{i j}\right|^{2}, \\
\mathcal{C}_{i j}= & 2 \max \left(0,\left|H_{i j}\right|-\sqrt{A_{i j} D_{i j}}\right), \\
L E_{i j} \geq & 2\left(\sqrt{A_{i j} D_{i j}}+\sqrt{B_{i j} C_{i j}}\right), \\
L E_{i j} \leq & \max \left(4\left|A_{i j} D_{i j}-B_{i j} C_{i j}\right|, 2 \operatorname{Re} H_{i j}\right) .
\end{array}
$$

A further simplification occurs for a state with exactly one down spin. Here, $A_{i j}$ the first diagonal element of the reduced density matrix vanishes.

Let us first consider an initial state with no entangled qubit pairs, given as

$$
\left|\psi_{u}(t=0)\right\rangle=|01111 \ldots\rangle .
$$

Under the time evolution with the above Hamiltonian, the down spin located at the first site propagates, through spin-1 magnon excitations. Denoting a state with the down spin at site $l$ by $|l\rangle$, the one-magnon eigenstates are given as $|q\rangle=\sum_{l} \phi_{l}(q)|l\rangle$. Here the onemagnon eigenfunction for a chain of $N$ qubits with open boundary conditions is

$$
\phi_{l}(q)=\sqrt{\frac{2}{N+1}} \sin (q l), q=\frac{\pi n}{N+1}, n=1, . . N,
$$

and the one-magnon eigenvalues are $\epsilon(q)=K \cos q$. The interaction term in the Hamiltonian does not figure in the magnon excitations in this sector with exactly one down spin. Now the time-evolved state at a time $t$ can be written as

$$
\left|\psi_{u}(t)\right\rangle=\sum_{l} \gamma_{l, 1}(t)|l\rangle
$$

where the time-dependent wave function is given by

$$
\gamma_{l, m}=\sum_{q} e^{-i \epsilon_{q} t} \phi_{l}(q) \phi_{m}^{\star}(q)
$$

The matrix elements of the reduced density matrix can be expressed in terms of the function $\gamma_{l, m}$. Let us consider the pair of qubits located at sites $i$ and $j$ We have $B=$ $\left|\gamma_{i, 1}\right|^{2}, C=\left|\gamma_{j, 1}\right|^{2}, D=1-B-C, H=\gamma_{i, 1} \gamma_{j, 1}^{\star}$.

Though we started with a state with no entanglement, in time pairs of qubits get entangled due to propagation of the down spin through magnon excitations. The different entanglement measures have been plotted in Fig. 1 as
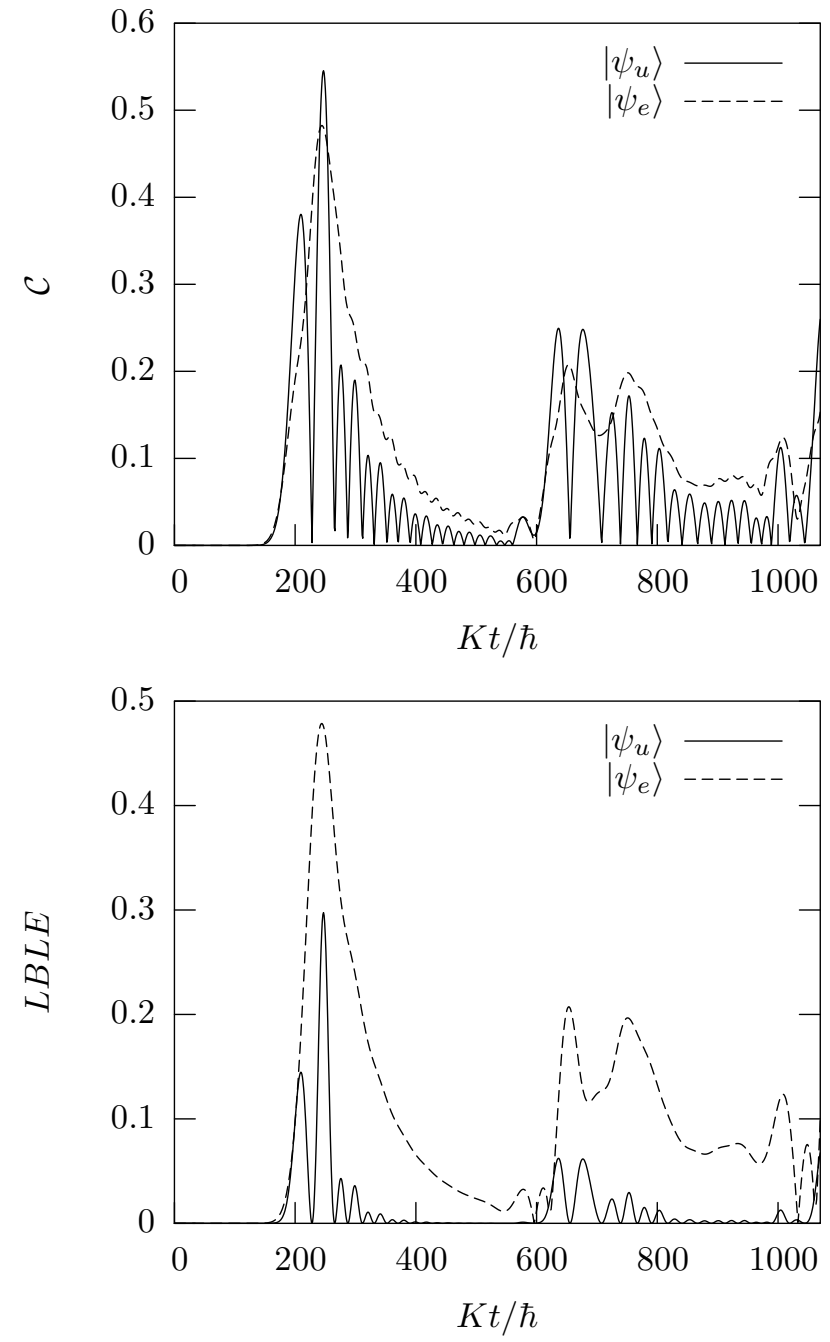

FIG. 2: The concurrence and LE measures of entanglement are plotted against time for unentangled and entangled initial states for the last pair of qubits for an open chain of $N=20$ qubits.

functions of time for the pair of qubits at the end of the chain (i.e. $i=N-1, j=N$ ) for a chain of $N=20$ qubits with open boundary conditions. The entanglement measures show time-dependent oscillatory behaviour, owing to the sinusoidal wave function. One interesting point is that the concurrence equals the upper bound on LE for one-magnon states.

The impurity measure, $M_{i j}$ is largest for almost all time, as it has contributions from two types of entanglements, namely the entanglement of this pair with the rest of the chain and the mutual entanglement between the two qubits. The concurrence is for the most part smaller than the impurity measure, but it is always greater than or equal to the lower bound on LE. The entanglement measures for various nearest neighbour pairs of qubits are shown for two different times, $K t / \hbar=50,150$. All the measures show peaks for some pair of qubits, the peak shifting with the time. 
It is important to note that all the measures have similar overall structures, for instance when the impurity of the pair of qubits is a local maximum, it is also the time when the pair is maximally entangled. Thus although purity (or lack of it) is not an entanglement measure, even in simple models as these there is a strong correlation between them. The LE as well as the concurrence share this property and hence we may say that usable entanglement is created with time at the end of the chain, even as the qubits become more mixed.

Let us now turn our attention to an initial state with the first pair of qubits maximally entangled, and the rest of qubits in state $|1\rangle$, given as

$$
\left|\psi_{e}(t=0)\right\rangle=\frac{1}{\sqrt{2}}|(01+10) 11111\rangle .
$$

The above state still has a definite number of down spins. Analogous to the unentangled state, we can write the state at a later time in this case as

$$
\left|\psi_{e}(t)\right\rangle=\frac{1}{\sqrt{2}} \sum_{l}\left(\gamma_{l, 1}+\gamma_{l, 2}\right)|l\rangle .
$$

Now the nonzero elements of the reduced density matrix are given by $D=1-B-C$, and

$$
\begin{array}{lc}
B_{i j}= & \frac{1}{2}\left|\gamma_{i, 1}+\gamma_{i, 2}\right|^{2}, \\
C_{i j}= & \frac{1}{2}\left|\gamma_{j, 1}+\gamma_{j, 2}\right|^{2}, \\
H_{i j}= & \frac{1}{2}\left(\gamma_{j, 1}^{\star}+\gamma_{j, 2}^{\star}\right)\left(\gamma_{i, 1}+\gamma_{i, 2}\right) .
\end{array}
$$

The envelope of the entanglement measures for the last pair of qubits as functions of time show similar behaviour as that of the unentangled state $\left|\psi_{u}\right\rangle$ shown in Fig. 1. We plot the different measures for the two cases of unentangled and entangled initial states in Fig. 2. The concurrence of the last two qubits for the initial state $\left|\psi_{e}\right\rangle$ is smoother than the case when the initial state is is totally unentangled, namely $\left|\psi_{u}\right\rangle$, in which case the entanglement shows rapid oscillations that repeatedly nearly vanish. The lower bound on LE, $(L B L E)$ shows up the difference between the two cases, even in the envelope. A part of the entanglement in LE for the last pair of qubits shown in the figure can be ascribed to the entanglement generated through dynamics, as in the case of the unentangled state, but a substantial part comes from the transport of initial entanglement of the first pair of qubits, through the Heisenberg dynamics.

In Fig. 3 we show a density plot of (the lower bound of) the localizable entanglement between neighbouring qubits, $L E_{l, l+1}(t)$, as a function of $l$, the location of the pair and the time, for a chain of $N=20$ qubits for an initial state with the first pair of qubits in a maximallyentangled state, that is $\left|\psi_{e}\right\rangle$. The brighter regions in the plot correspond to larger entanglement for the given pair at the given time. We can see the front of bright "entanglement" patch moving across the chain and reach the end linearly in time, getting reflected, and again moving back and forth. After one traversal the bright patch is

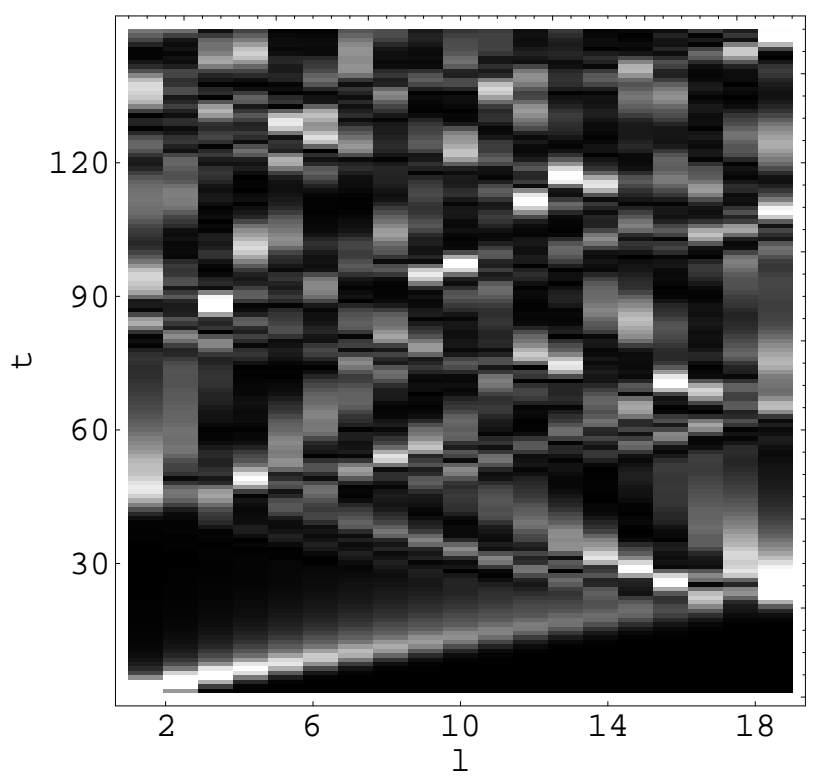

FIG. 3: $L B L E_{l, l+1}(t)$ is plotted as function of $l$ (along x-axis) and the time $t$, with brighter regions corresponding to larger entanglement, for a chain of $N=20$ qubits for the initial state $\left|\psi_{e}\right\rangle$. The bright patch travels across the chain to reach the end.

not so clearly seen, as entanglement begins to be shared by more than one neighbouring pair of qubits, simultaneously. For the first passage across the chain, the location of the patch at time $t$ is, $l=v t$, where $v=K$ is the velocity of the front. For a nonzero entanglement at a distance $l$, the pair of qubits located at $l, l+1$ have to first become a part of a mixed state. This happens through the transport of the down spin located at the first site via one-magnon excitations. The time scale associated with the magnons is $\tau=1 / K$, and $v=1 / \tau$.

In conclusion, we have investigated the dynamics of Heisenberg-XY spin chain using the various pairwise entanglement measures. Initial states with no entangled pairs of qubits, and with exactly one pair of entangled qubits are studied through the time-evolution, and the their dynamics of entanglement is juxtaposed. We have shown that the localizable entanglement bound captures the essential features of entanglement transport, and contrasts better the dynamics of a state with no initiallyentangled pair of qubits from the dynamics of a state with a pair of qubits maximally entangled. The entanglement transport occurs through magnon excitations, and the first passage to the end of the chain of the pair entanglement front occurs with a uniform rate. 
1 M. A. Nielsen and I. L. Chuang, Quantum Computation and Quantum Information (Cambridge University Press, Cambridge, 2000).

2 A. Peres, Quantum Theory: Concepts and Methods (Kluwer Academic, Dordrecht, 1993).

3 W. K. Wootters, Phys. Rev. Lett. 80, 2245 (1998).

4 F. Verstraete, M. Popp, and J. I. Cirac, Phys. Rev. Lett. 92, 027901 (2004).

5 P. Zanardi, Phys. Rev. A65, 042101 (2002).

6 A. Lakshminarayan and V. Subrahmanyam, Phys. Rev.
A67, 052304 (2003).

7 V. Subrahmanyam, Phys. Rev. A69, 022311 (2004).

8 S. Bose, Phys. Rev. Lett. 91, 207901 (2003).

9 V. Subrahmanyam, Phys. Rev. A69, 034304 (2004).

10 T. Osborne and N. Linden, Phys. Rev. A69, 052315 (2004).

11 B. -Q. Jin and V. E. Korepin, Phys. Rev. A69, 062314 (2004). 